\title{
WHAT THE FINITIZATION PROBLEM IS NOT
}

\author{
A. SIMON \\ Mathematical Institute of the Hungarian Academy of Sciences \\ P.O. Box 127, Budapest, H-1364, Hungary \\ E-mail:H3761SIM@ELLA.HU
}

0. Introduction. The finitization problem is one of the problems considered important in algebraic logic. Since it is often misunderstood, it seems desirable to try to develop a better understanding of what it is and what it is not about.

The finitization problem is (at least partly) motivated by the following observations (1)-(3): (1) The natural algebraic counterpart of propositional logic is the variety of Boolean algebras which is axiomatizable by finitely many equations. (2) The natural algebraic counterpart of first-order logic $L_{\omega \omega}$ is the variety RCA of representable cylindric algebras which is very far from being axiomatizable by a finite schema of equations, cf. [HMT], Theorem 4.1.3, and Andréka [A91]. (3) The algebraic counterpart of $L_{\omega \omega}$ without equality is the variety RQPA $\omega$ of representable quasi- $p$ olyadic algebras which is also not axiomatizable by a finite schema, see Sain-Thompson [ST]. (Similar negative results apply to the finitevariable fragments of $L_{\omega \omega}$. The algebraic counterparts here are finite-dimensional representable cylindric or polyadic algebras and relation algebras.) The negative results (2) and (3) do have purely logical consequences motivating the (nonalgebraic) logician to look into the question, cf. e.g. §4 of Sain [S87], Simon [S90], [S91], Venema [V90], [V92], Németi [N91], but see also the present Appendix A.

Observations (2) and (3) above are in contrast with (1). So there seems to be a sharp contrast between the behaviour of propositional logic and quantifier logics. It is natural to ask whether the nonfinitizability in (2) and (3) is an unavoidable

1991 Mathematics Subject Classification: 03G15, 03B99, 03C99.

Research supported by Hungarian National Foundation for Scientific Research grants Nos. 1911 and 2258 .

Thanks to Hajnal Andréka, Ivo Düntsch, Ágnes Kurucz, Szabolcs Mikulás and István Németi.

The paper is in final form and no version of it will be published elsewhere. 
consequence of having quantification in our logics, or perhaps the negative results (2), (3) are only consequences of "historical accidents" which have led to the generally accepted formulation of $L_{\omega \omega}$. A more tangible formulation of this question is Problem 0 below. But first we need a definition.

In this paper we let the algebraic counterpart $\operatorname{Alg}\left(L_{1}\right)$ of the logic $L_{1}$ be the quasivariety generated by the class $\operatorname{LT}\left(L_{1}\right)$ of semantical Lindenbaum-Tarski algebras of theories of $L_{1}$, where (i) and (ii) below are important to keep in mind: (i) The operations of $\operatorname{Alg}\left(L_{1}\right)$ are exactly the logical connectives of $L_{1}$. (Note that, in general, if $C$ is an $n$-ary logical connective, then $C\left(\varphi_{1}, \ldots, \varphi_{n}\right)$ is a formula whenever $\varphi_{1}, \ldots, \varphi_{n}$ are formulas. So for example $\exists$ is not a logical connective in $L_{\omega \omega}$, but $\exists x$ is one, for each individual variable $x$.) (ii) When forming the Lindenbaum-Tarski algebra of a theory, say $T$ of $L_{1}$, we factor out with the semantical equivalence $\equiv_{T}$ defined by $\varphi \equiv_{T} \psi$ iff $(T \vDash \varphi \leftrightarrow \psi)$, for every pair $\varphi, \psi$ of $L_{1}$-formulas $\left(^{1}\right)$.

Problem 0. Is there a logic $L_{1}$ satisfying conditions (1)-(4) below?

(1) $L_{1}$ has the same models as $L_{\omega \omega}$.

(2) The logical connectives of $L_{\omega \omega}$ are definable in $L_{1}$.

(3) $L_{1}$ satisfies the most basic axiom of Abstract Model Theory (cf. e.g. Barwise-Feferman $[\mathrm{BF}])$, namely the satisfaction relation of $L_{1}$ is invariant under isomorphisms of models.

(4) The algebraic counterpart $\operatorname{Alg}\left(L_{1}\right)$ of $L_{1}$ is a finitely axiomatizable variety.

We note that though Problem 0 is only an approximation of the finitization problem, it seems inevitable that any solution of the finitization problem should solve Problem 0, too. For a careful recent formulation of (different versions of) the finitization problem see Németi $[\mathrm{N} 91]\left({ }^{2}\right)$. Appendix A herein contains further information on the problem not available in [N91]. The problem was published in Henkin-Monk [HM], cf. also Monk [M70]. The purely logical form was presented by Henkin to Andréka, Németi and Sain at the 1987 Algebraic Logic Conference in Asilomar (cf. Problem 2 in Appendix A herein).

As pointed out e.g. in Maddux [M], the problem itself is fairly complex and is easy to misunderstand. As we said before, here we will try to develop a better understanding of what the problem is and what it is not about. To this end we will look at a very natural-looking interpretation, one which comes to one's mind very easily when reading about finitization. We will see that no matter

$\left({ }^{1}\right)$ The present definition of $\operatorname{Alg}\left(L_{1}\right)$ is in the spirit of the (more careful) definitions in the basic literature, cf. [HMT], §5.6, [ANS] or Blok-Pigozzi [BP]. (Refined versions of our $\operatorname{Alg}\left(L_{1}\right)$ are implicit in the present Appendix A.)

$\left(^{2}\right)$ Cf. the following parts of that paper: Remark 2, the last two pages of $\S 4$, especially Problems 2.1, 2.2, the part beginning with Theorem 16 and containing item (5.1) and Problem 3 in $\S 7(9)$. 
how tempting, this is still not strong enough to be a reasonable version of the finitization problem.

Consider

TASK (*). Find a finitely axiomatizable variety $V$ and a computable function $F$ mapping all first-order formulas to the set of equations in the language of $V$ such that the following holds.

For every first-order formula $\varphi$, we have

$$
\vDash \varphi \quad \text { iff } \quad V \vDash F(\varphi) .
$$

An additional requirement is that

(**) F should be "semantically satisfactory" in the following "one way" sense: There is a function Alg associating with every model $\mathfrak{M}$ an algebra $\operatorname{Alg}(\mathfrak{M}) \in V$ such that for every formula $\varphi$ of $L_{\omega \omega}$, the meaning of $\varphi$ in $\mathfrak{M}$ can be identified somehow $\left({ }^{3}\right)$ with the meaning of $F(\varphi)$ in $\operatorname{Alg}(\mathfrak{M})$.

We call (**) a "one way" condition because the elements of $V$ are not required to be translatable back to models or to anything obtained from models. By contrast, every element of $\mathrm{RCA}_{\omega}$ corresponds to a set of models (cf. [HMT], 4.3.9, 4.3.52 and in general $\S 4.3$ therein). So in the $\mathrm{RCA}_{\omega}$ case we have a stronger, "two way" semantical connection.

Our reason to formulate Task $(*)$ when trying to clarify the finitization problem is the following:

Incarnations of Task $(*)$ with or without $(* *)$ come up frequently (as e.g. reasonable variants) in connection with the finitization problem in the literature and in related discussions. Also, in view of our having motivated the finitization problem by listing items (1)-(3), Task (*) looks quite appealing, because its conclusion holds for propositional logic; therefore one is tempted to think that what we are asking for in Task $(*)$ is the property of propositional logic which has been lost when we moved to $L_{\omega \omega}$. That Task $(*)$ is not a reasonable variant of the finitization problem is perhaps best shown by actually solving this task and then contemplating what logical significance the solution has. In particular, whether it solves the above mentioned logical problem, which, as far as we know, motivated at least one of the originators of the finitization problem. (This logical problem will be recalled as Problem 2 in Appendix A, but Problem 0 above is a good approximation of it.) We will find that the answer to this question is in the negative, and at the end of this paper we will try to spell out the reason why. In $\S 1$ below we show that Task $(*)$ admits almost trivially easy solutions, while in $\S 2$ we do the same for the stronger version of Task $(*)$ reinforced with condition $(* *)$. In our opinion, the solutions we give are of the difficulty of a perhaps tedious, but

$\left({ }^{3}\right)$ For example, if we take $V$ to be $\mathrm{RCA}_{\omega}$, then we can choose $F(\varphi)$ to be of the form $F_{1}(\varphi)=1$. To a model $\mathfrak{M}$ there corresponds an algebra $\operatorname{Alg}(\mathfrak{M}) \in \mathrm{RCA}_{\omega}$ together with a valuation $k$ of the (algebraic) variables of $F_{1}(\varphi)$ such that the value of $F_{1}(\varphi)$ in $\operatorname{Alg}(\mathfrak{M})$ at the valuation $k$ coincides with $\left\{q \in{ }^{\omega} M: \mathfrak{M} \vDash \varphi[q]\right\}$. See [HMT], $\S 4.3$ for details. 
deterministic exercise for someone familiar with the pre-1960 logic (or, in the case of $(* *)$, algebraic logic) background. Consequently, to us it seems quite unlikely that this task could be the well known (and publicized) finitization problem.

We should point out that the purpose of this paper is entirely different from solving Task $(*)$. Solutions for it have been known for quite a while before this paper was conceived. E.g. Maddux [M], Theorem 23, also contains a solution of Task $(*)$ without $(* *)$ and Theorems 21,22 therein together also solve Task $(*)$ with $(* *)$. Sometimes Veloso-Haeberer $[\mathrm{VH}]$ is also quoted in this connection. (Actually, a letter Tarski wrote to Quine in 1942 seems to imply that he, and therefore, in our opinion, the authors of [HMT], were aware of solvability of Task $(*)$ all the time while working on [HMT] and on related papers, cf. Tarski-Givant [TG], p. 165.)

1. Solution of Task $(*)$ (via elementary recursion theory). In this section we will show that without $(* *)$ there is a very easy solution, almost as easy as the following. Let $V$ be any finitely axiomatizable variety and let $F(\varphi)$ be $x=x$ if $\varphi$ is valid and $x=y$ otherwise. This obviously works (and is obviously quite useless). The only reason why this is not yet a solution of Task $(*)$ without $(* *)$ is that $F$ is not computable. But by using Gödel's completeness theorem and a few similarly old facts this can be easily corrected so that the idea remains the same. Here is one way to do that:

By Gödel's completeness theorem the validity problem of $L_{\omega \omega}$ is reducible to the halting problem of a Turing machine $M$. By the standard textbook proof (originating with Post from the 1940's) of the unsolvability of the word problem of semigroups, the halting problem of any Turing machine is reducible to the validity of quasiequations in the variety Smg of semigroups. So the only work left to be done is reducing validity of quasiequations in Smg to validity of equations in a perhaps different (finitely axiomatizable) variety. For this, there are many easy, well-known methods. One possible way is to add a new discriminator function to Smg. Another one is elaborated below.

First we recall Post's lemma mentioned above (see [D] (in the Handbook of Mathematical Logic), Theorem 2.4 and Lemma 3.2, for a slightly different but equivalent formulation). Let $\mathrm{Fm}$ be the set of formulas of $L_{\omega \omega}$ and let $T$ be any Turing machine taking elements of Fm as inputs. Let Eqlang(Smg) be the set of equations in the language of semigroups.

Lemma (Post, 1947). There is a finite set $E \subseteq \operatorname{Eqlang}(\mathrm{Smg})$ and a computable function $f: \mathrm{Fm} \rightarrow \operatorname{Eqlang}(\mathrm{Smg})$ such that for all $\varphi \in \mathrm{Fm}$

$$
T \text { halts for the input } \varphi \Leftrightarrow \operatorname{Smg} \vDash(\bigwedge E) \rightarrow f(\varphi) \text {. }
$$

(Note that $(\bigwedge E) \rightarrow f(\varphi)$ is a quasiequation.)

First we apply Post's lemma to the machine $M$ fixed above. Next we reduce validity of quasiequations to that of equations by expanding the variety Smg 
with new constant symbols. Let $\bar{x}=\left\langle x_{0}, \ldots, x_{k}\right\rangle$ be the sequence of all variables occurring in $E$. Let $\bar{c}=\left\langle c_{0}, \ldots, c_{k}\right\rangle$ be a sequence of new constant symbols. We add $\bar{c}$ to the language of Smg, obtaining $\mathrm{Smg}^{+}$. Let $\mathrm{Smg}^{E}$ be the subvariety of $\mathrm{Smg}^{+}$defined by $E(\bar{c} / \bar{x})$, where $(\bar{c} / \bar{x})$ is the operation of substituting $c_{i}$ for all occurrences of $x_{i}$ for each $i \leq k$. Clearly

$$
\mathrm{Smg} \vDash(\bigwedge E) \rightarrow f(\varphi) \Leftrightarrow \operatorname{Smg}^{E} \vDash f(\varphi)(\bar{c} / \bar{x}) .
$$

Now, Post's lemma implies

$$
\vDash \varphi \Leftrightarrow M \text { halts for } \varphi \Leftrightarrow \operatorname{Smg}^{E} \vDash f(\varphi)(\bar{c} / \bar{x}),
$$

for all $\varphi \in \mathrm{Fm}$.

Let $F(\varphi) \stackrel{\text { def }}{=} f(\varphi)(\bar{c} / \bar{x})$. Then

Corollary 0. For all $\varphi \in$ Fm we have

$$
\vDash \varphi \Leftrightarrow \operatorname{Smg}^{E} \vDash F(\varphi) .
$$

Further, $\mathrm{Smg}^{E}$ is a finitely axiomatizable variety and $F$ is a computable function sending formulas to equations.

Proof. Immediate by statement (0) above (i.e. by Post's lemma and our having replaced variables with constant symbols).

Corollary 0 above is clearly a solution of Task $(*)$. At the same time, in our opinion, it is not much more than a simple reformulation of Post's lemma. For the convenience of those readers who are not familiar with (the above quoted quasiequational form of) Post's lemma, we give a slightly different solution in Appendix B, in which all the quoted lemmas will be used exactly in the form in which they are stated in $[\mathrm{D}]$.

R e m a rk. Before looking at a different kind of solution for Task (*) (based on a result of Henkin from 1955 instead of Post's 1947 lemma) we would like to mention that the present solution, i.e. Corollary 0 (or, equivalently, Theorem B.0 in Appendix B), can be transformed to one satisfying (**), too. The reason why this is possible is that in $(* *)$ semantical translatability is required in one direction (logic $\rightarrow$ algebra) only. Hint: Use the fact (mentioned in $\S 0$ ) that RCA $\mathrm{A}_{\omega}$ satisfies $(* *)$. Start out with $\mathrm{Smg}^{E}$ and $F$ as in Corollary 0. Let Smg' be obtained from Smg ${ }^{E}$ by adding all the operations of $\mathrm{RCA}_{\omega}$ to $\mathrm{Smg}^{E}$ and postulating nothing about them. Define the function Alg mapping models of $L_{\omega \omega}$ into (the "RCA ${ }_{\omega}$ part" of) Smg' the standard way (described in [HMT], §4.3, and already alluded to in a footnote in $\S 0$ herein) and let the semigroup structure of $\operatorname{Alg}(\mathfrak{M})$ be idempotent, i.e. let $x y=x$. Note that at this point Corollary 0 is still true for $\mathrm{Smg}^{\prime}$ in place of $\mathrm{Smg}^{E}$. Modify $F$ in such a way that the meaning of $F(\varphi)$ in $\operatorname{Alg}(\mathfrak{M})$ comes out to be the same as in [HMT], $\S 4.3$, but Corollary 0 still holds for Smg and the new $F$ in place of $\mathrm{Smg}^{E}$ and the old $F$. We do not claim that the reader attempting to implement this hint will have no difficulties, but those are 
not very hard to overcome by using [HMT] and [BS]. We do not go into details since a solution of Task $(*)$ with $(* *)$ is already available in $\S 2$.

2. Semantically satisfactory solution of Task $(*)$ (via elementary algebraic logic). First we would like to make some comments on why we think that the problem we are about to solve is really just an exercise. Here is an outline of a possible solution, where in each step we give reasons why we think the step in question is completely routine.

PLAN. Step 0. The obvious starting point is looking into classical textbooks (like $[\mathrm{HMT}]$ ) on algebraizations of $L_{\omega \omega}$. There we find that the variety RCA of representable cylindric algebras is related to $L_{\omega \omega}$ the same way BA is related to propositional logic; namely there is a computable function $\tau \mu^{+}$associating RCA-terms with formulas of $L_{\omega \omega}$ such that

$$
\vDash \varphi \quad \text { iff } \quad \operatorname{RCA}_{\omega} \vDash \tau \mu^{+}(\varphi)=1
$$

for every first order formula $\varphi$, cf. [HMT], $4.3 .61\left(^{4}\right)$.

$\mathrm{S}$ tep 1 . The only problem is that $\mathrm{RCA}_{\omega}$ is not a finitely axiomatizable variety. Let us see how standard textbooks bypass this problem in logical applications of cylindric algebra (CA from now on) theory:

There is a finite schema axiomatizable variety $C_{\omega}\left(\supset \mathrm{RCA}_{\omega}\right)$ such that $R C A_{\omega}$ is easily identifiable in $\mathrm{CA}_{\omega}$ by simple properties called conditions for representability. (We will see later that moving from finite schema to finitely many axioms is no problem under the permissiveness of Task (*).) One of these properties (published by Henkin in 1955 (cf. [H]) and extensively discussed in Part I of [HMT] $)$ is called neat embeddability, and it says that a CA is in RCA iff we can add new (elements and) operations to it such that the expanded system satisfies some equations. Note that in Task $(*)$ we are allowed to add new operations that are permitted to behave in arbitrarily strange ways $\left({ }^{5}\right)$. Let us see how we can use this here.

What would go wrong if we simply replaced RCA with CA in $(\dagger)$ above? The problem is that there are $\varphi$ and $\mathfrak{A} \in \mathrm{CA}_{\omega}$ such that $\vDash \varphi$ but $\mathfrak{A} \not \models \tau \mu^{+}(\varphi)=1$ exactly because $\mathfrak{A} \notin \mathrm{RCA}_{\omega}$. To avoid this we do not require representability of the whole of $\mathfrak{A}$, but only of that part of $\mathfrak{A}$ where $\tau \mu^{+}(\varphi)$ is computed. According to

$\left({ }^{4}\right)$ Our $\tau \mu^{+}$is the "equational version" of the quasi-equation in [HMT], 4.3.61(ii). This is easy to obtain, namely, using the notation of the quoted item, let $\Gamma_{j} \stackrel{\text { def }}{=} H \backslash \varrho(j)$ for each $j \in G$. Obviously, $\Gamma_{j}$ is finite. Replace each occurrence of the algebraic variable $v_{j}$ in $\tau \mu^{\prime}(\varphi)$ with the term $\mathrm{c}_{\left(\Gamma_{j}\right)} v_{j}$. Execute this for all $j \in G$. The term so obtained is $\tau \mu^{+}(\varphi)$ and the quoted theorem states the above $(\dagger)$ for this $\tau \mu^{+}$.

$\left({ }^{5}\right)$ The standard terminology for this property is pseudo-axiomatizability. (A class $K$ is called pseudo-axiomatizable (or pseudo-elementary) iff it is a reduct of a finitely axiomatizable class of structures, cf. e.g. [HMT], 0.5.21.) It has been known for a long time in the classical logic literature that it is very easy to obtain pseudo-axiomatizibility results (cf. eg. Craig-Vaught $[\mathrm{CV}]$ and Fact 1 in [N91]). We will utilize this phenomenon several times below. 
$[\mathrm{H}]$ this can be achieved by adding new elements to $\mathfrak{A}$ obtaining $\mathfrak{A}^{+} \supseteq \mathfrak{A}$, adding new operations to $\mathfrak{A}^{+}$and postulating that some simple equations $\left(c_{i} a=a\right)$ hold for the new operations $\mathrm{c}_{i}(i \notin \omega)$ on the old elements $a \in A$. This expanded version $\mathfrak{A}^{\mathrm{e}}$ of $\boldsymbol{\mathfrak { A }}^{+}$is called a $\mathrm{CA}_{\omega+\omega}$, where the " $+\omega$ " refers to the new operations. Technically, $\boldsymbol{\mathfrak { A }}^{+}$is a reduct of $\boldsymbol{\mathfrak { A }}^{\mathrm{e}} \in \mathrm{CA}_{\omega+\omega}$. What we want to achieve is that $\tau \mu^{+}(\varphi)$ should be computed in the old part of $\boldsymbol{A}^{\mathrm{e}}$, corresponding to $A$. This is very easy to arrange because Task $(*)$ permits us to introduce new operation symbols. So, we add a new predicate symbol $A$ to the language of $\mathrm{CA}_{\omega+\omega}$ and postulate that $\forall x\left(A(x) \rightarrow \mathrm{c}_{i}(x)=x\right)$ for each $i \notin \omega$. Further, we restrict the variables in $\tau \mu^{+}(\varphi)$ to range over $A$. To make all this equational is a very old exercise, namely we use a unary function symbol $a$ the range of which codes $A$. Then the previous postulate reads as $\mathrm{c}_{i}(a(x))=a(x)$, and each variable $x$ in $\tau \mu^{+}(\varphi)$ is replaced with $a(x)$. From now on we will skip this obvious trick of "equationalizing" unary predicates. Let $\mathrm{CA}^{+}$be the variety obtained from $\mathrm{CA}_{\omega+\omega}$ by adding the new operation $a$ and the above equations.

Now if we compute the new version of $\tau \mu^{+}(\varphi)$ in our CA $^{+}$'s, the elements involved are all in the subalgebra generated by the subset $A$. But this subalgebra is representable according to $[\mathrm{H}]$ since we required $A$ to satisfy the neat embeddability condition $\left(\forall x\left(A(x) \rightarrow \mathrm{c}_{i}(x)=x\right)\right.$ for $\left.i \notin \omega\right)$. Since $\tau \mu^{+}(\varphi)$ is computed in this subalgebra, by $(\dagger)$ we have the "hard" direction $\left(\vDash \varphi\right.$ implies $\left.\mathrm{CA}^{+} \vDash \tau \mu^{+}(\varphi)=1\right)$ in

$$
\vDash \varphi \quad \text { iff } \quad \mathrm{CA}^{+} \vDash \tau \mu^{+}(\varphi)=1 .
$$

The other direction is obvious.

Step 2. The only reason why $(\dagger \dagger)$ is not yet a solution of Task $(*)$ is that the latter asks for a finitely axiomatizable, and not a finite schema axiomatizable class. Is there a well known trick in classical textbooks on universal algebra for reducing a finite schema of equations to finitely many equations? The answer is yes, switch to two-sorted algebras (a typical example is the class of modules over some ring $\left.\left({ }^{6}\right)\right)$. Using this trick we arrive at two-sorted algebras $C A^{2}$, where one sort is the "CA sort" and the other is the index sort. Then $C^{2}$ has finitely many operations. Furthermore, it is defined by finitely many equations since all we have to postulate about the index sort is that it is infinite (a typical pseudoaxiomatizable property) and we can add a discriminator function to be able to express the axioms equationally (cf. Burris-Sankappanavar $[\mathrm{BS}]$ ). So, by (††) we have

$$
\vDash \varphi \quad \text { iff } \quad \mathrm{CA}^{2} \vDash \tau \mu^{+}(\varphi)=1
$$

for the finitely axiomatizable class $C A^{2}$.

$\left({ }^{6}\right)$ Or vector spaces; cf. the textbook [B86], $§ 1.2 .1$ and the references therein. 
Step 3. The only objection to claiming that $(\dagger \dagger \dagger)$ is a solution of Task $(*)$ might be that $\mathrm{CA}^{2}$ is two-sorted. Again, classical logic textbooks explain how to turn two-sorted structures to one-sorted ones: add two new unary relation symbols, say $B$ and $I$, to the language of $C A^{2}$ and use them instead of the $C A$ sort and the index sort, respectively. The old axioms about the index sort are now axioms about those elements of the algebra which are in $I$, and similarly for the other sort. So, by $(\dagger \dagger \dagger)$, we arrived at a one-sorted, finitely axiomatized variety $\mathrm{CA}^{1}$ with

$$
\vDash \varphi \quad \text { iff } \quad \mathrm{CA}^{1} \vDash \tau \mu^{+}(\varphi)=1,
$$

that is, we solved Task $(*)$.

Let us turn to condition $(* *)$ briefly. The original translating function $\tau \mu^{+}$is certainly satisfactory (cf. [HMT], $§ 4.3$, especially item (1) in the proof of 4.3.61 on p. 173). In our procedure above we did not change the basic parts of CA's which show up in the translation of formulas. Consequently, the nice semantic properties of the original translating function $\tau \mu^{\prime}$ remained untouched. This will indeed be checked in Theorem 2.11 (admitting a trivial proof) close to the end of this paper.

Note that what we required no ingenuity whatsoever, just looking into basic textbooks on the subject.

For completeness we give a detailed proof below (which does not literally follow the above plan, simply because we did not want to have too many symbols and axioms). But the reader is invited to skip this proof and fill in the details of the above plan according to his/her taste, and resume reading after Lemma 2.10.

Soon we will introduce the class SCA (where the S stands for any of the s's in "one-sorted CA's simulating two-sorted ones"), another finitely based variety, and a translating function $\bar{F}$ (being more intuitive than $F$ of the previous section from the semantical point of view). First we formulate a theorem which says that we have another solution of Task $(*)$. See 2.5 and 2.9 below for the definition of SCA and $\bar{F}$. After proving Theorem 2.0 we show that this solution satisfies condition $(* *)$, too, see Theorem 2.11.

TheOREM 2.0. For $\varphi$, a first-order formula,

$$
\vDash \varphi \Leftrightarrow \mathrm{SCA} \vDash \bar{F}(\varphi) .
$$

Further, $\bar{F}$ is computable and SCA is a finitely based variety.

Pr o of. By Lemmas 2.4 and 2.10 below.

DEFINITION 2.1.

$$
\mathfrak{A}=\left\langle A,+,-, 1, \mathrm{c}_{i}, \mathrm{~d}_{i j}, \mathrm{k}_{m}\right\rangle_{i, j \in \mathbb{Z}, m \in \omega} \in \mathrm{CA}_{\mathbb{Z}}^{k}
$$

iff $\boldsymbol{\mathfrak { A }} \vDash\left(\mathrm{C}_{0}\right)-\left(\mathrm{C}_{8}\right)$ where $\left(\mathrm{C}_{0}\right)-\left(\mathrm{C}_{7}\right)$ are as in $[\mathrm{HMT}], 1.1 .1$, and

$\left(\mathrm{C}_{8}\right) \quad \mathrm{c}_{i} \mathrm{k}_{m}=\mathrm{k}_{m} \quad$ for all $i<0 \leq m$. 
Definition 2.2. If $\varphi$ is a first-order formula then let

$$
H(\varphi) \stackrel{\text { def }}{=}\left\{i: v_{i} \text { occurs in } \varphi\right\} .
$$

Restricted first order formulas were defined in [HMT], $\S 4.3$ (p. 152), where it was also shown that every formula has an equivalent restricted "normal form". Throughout, $n=\{k: k<n\}$ for $n \in \omega$.

DEFINITION 2.3. Define the function $F$ mapping first-order restricted formulas to constant (i.e. variable-free) $\mathrm{CA}_{\mathbb{Z}}^{k}$-terms as follows. For $\varphi$, a restricted first-order formula, let $F(\varphi) \stackrel{\text { def }}{=} F_{\varphi}(\varphi)$, where $F_{\varphi}$ is defined by recursion on the subformulas of $\varphi$ by the following clauses:

$$
\begin{aligned}
& F_{\varphi}\left(\mathbf{R}_{j} v_{0} \ldots v_{n-1}\right)=\mathrm{c}_{(H(\varphi) \backslash n)} \mathrm{k}_{j}, \\
& F_{\varphi}\left(v_{i}=v_{j}\right)=\mathrm{d}_{i j}, \\
& F_{\varphi}\left(\psi_{1} \wedge \psi_{2}\right)=F_{\varphi}\left(\psi_{1}\right) \cdot F_{\varphi}\left(\psi_{2}\right), \\
& F_{\varphi}(\neg \psi)=-F_{\varphi}(\psi) \\
& F_{\varphi}\left(\exists v_{i} \psi\right)=c_{i} F_{\varphi}(\psi)
\end{aligned}
$$

where $\mathrm{c}_{(\Gamma)} x \stackrel{\text { def }}{=} \mathrm{c}_{i_{1}} \ldots \mathrm{c}_{i_{m}} x$ if $\Gamma=\left\{i_{1}, \ldots, i_{m}\right\}$, just as in [HMT], 1.7.1.

Note that $F$ is computable and so is the function giving the restricted equivalent of an arbitrary formula. So, with a little abuse of notation, we shall write $F(\varphi)$ even if $\varphi$ is not a restricted formula.

LEMMA 2.4. $\vDash \varphi$ iff $\mathrm{CA}_{\mathbb{Z}}^{k} \vDash F(\varphi)=1$.

Proof. We can assume that $\varphi$ is restricted. Then by [HMT], 4.3.61, it is enough to show that

$$
\begin{aligned}
\mathrm{CA}_{\mathbb{Z}}^{k} \vDash F(\varphi)=1 & \Leftrightarrow \\
\operatorname{RCA}_{\omega} & \vDash \bigwedge\left\{c_{i} v_{j}=v_{j}: \mathbf{R}_{j} \in \varphi, i \in H(\varphi) \backslash \varrho(j)\right\} \rightarrow \tau \mu^{\prime}(\varphi)=1
\end{aligned}
$$

where $\varrho(j)$ is the rank of $\mathbf{R}_{j}$, " $\mathbf{R}_{j} \in \varphi$ " abbreviates that the relation symbol $\mathbf{R}_{j}$ occurs in $\varphi$, and $\tau \mu^{\prime}$ is defined in [HMT], 4.3.60.

$(\Rightarrow)$ Suppose that $\operatorname{RCA}_{\omega} \not \models \bigwedge\left\{c_{i} v_{j}=v_{j}: \mathbf{R}_{j} \in \varphi, i \in H(\varphi) \backslash \varrho(j)\right\} \rightarrow$ $\tau \mu^{\prime}(\varphi)=1$ and let $\mathfrak{A} \in \mathrm{RCA}_{\omega}$ and $V: \omega \rightarrow A$ be such that $\mathrm{c}_{i}^{\mathfrak{A}} V(j)=V(j)$ if $\mathbf{R}_{j} \in \varphi$ and $i \in H(\varphi) \backslash \varrho(j)$, but $\left(\tau \mu^{\prime} \varphi\right)^{\mathfrak{A}}[V] \neq 1^{\mathfrak{A}}$. Since $\mathrm{RCA}_{\omega}=\mathbf{S} \mathrm{Nr}_{\omega} \mathrm{CA}_{\mathbb{Z}}$ by [HMT], 3.2.10 $\left({ }^{7}\right)$, there is a $\mathfrak{B} \in \mathrm{CA}_{\mathbb{Z}}$ with $\mathfrak{A} \leq \mathfrak{N r}_{\omega} \mathfrak{B}$. Let $\mathfrak{B}^{+} \stackrel{\text { def }}{=}\left\langle\boldsymbol{B}, \mathrm{k}_{j}\right\rangle_{j \in \omega}$, where $\mathrm{k}_{j}^{\mathfrak{B}^{+}} \stackrel{\text { def }}{=} V(j)$ if $j \in \omega$. Then $\mathfrak{B}^{+} \in \mathrm{CA}_{\mathbb{Z}}^{k}$, that is, it satisfies $\left(\mathrm{C}_{8}\right)$, since $V(j) \in A \subseteq C l_{\mathbb{Z} \backslash \omega} \mathfrak{B}$.

Next we show that $F(\varphi)^{\mathfrak{B}^{+}}=\left(\tau \mu^{\prime}(\varphi)\right)^{\mathfrak{A}}[V]$ by induction on the subformulas of $\varphi$. This will complete this part of the proof since $1^{\mathfrak{B}^{+}}=1^{\mathfrak{A}} \neq\left(\tau \mu^{\prime}(\varphi)\right)^{\mathfrak{A}}[V]$.

$\left({ }^{7}\right) \mathrm{CA}_{\mathbb{Z}}$ is the class $\mathrm{CA}_{\omega+\omega}$ modulo renaming the indices. As we can learn e.g. from [MLn], there is no reason to stick to ordinals as possible index sets. 
Suppose that $\varrho(j)=n$ and $\mathbf{R}_{j} v_{0} \ldots v_{n-1}$ is a subformula of $\varphi$. Then

$$
\begin{aligned}
\left(\tau \mu^{\prime} \mathbf{R}_{j} v_{0} \ldots v_{n-1}\right)^{\mathfrak{A}}[V] & =V(j)=\mathrm{c}_{(H(\varphi) \backslash n)}^{\mathfrak{A}} V(j)=\mathrm{c}_{(H(\varphi) \backslash n)}^{\mathfrak{B}^{+}} V(j) \\
& =\left(\mathrm{c}_{(H(\varphi) \backslash n)} \mathrm{k}_{j}\right)^{\mathfrak{B}^{+}}=\left(F_{\varphi}\left(\mathbf{R}_{j} v_{0} \ldots v_{n-1}\right)\right)^{\mathfrak{B}^{+}} .
\end{aligned}
$$

The case of $v_{i}=v_{j}$ and the induction steps are immediate since here the definition of $F_{\varphi}$ parallels that of $\tau \mu^{\prime}$ and the operations of $\mathfrak{A}$ are restrictions of the corresponding operations of $\mathfrak{B}^{+}$.

$(\Leftarrow)$ Suppose that $\mathrm{CA}_{\mathbb{Z}}^{k} \not \models F(\varphi)=1$ and let $\mathfrak{B}^{+} \in \mathrm{CA}_{\mathbb{Z}}^{k}$ be such that $F(\varphi)^{\mathfrak{B}^{+}} \neq$ $1^{\mathfrak{B}^{+}}$. Let $\mathfrak{B}$ be the $\mathrm{CA}_{\mathbb{Z}}$-reduct of $\mathfrak{B}^{+}$and let $\mathfrak{A} \stackrel{\text { def }}{=} \mathfrak{N} \mathfrak{r}_{\omega} \mathfrak{B}$. Then, by [HMT], $3.2 .10, \mathfrak{A} \in \mathrm{RCA}_{\omega}$. Define the valuation $V: \omega \rightarrow A$ by

$$
V(j) \stackrel{\text { def }}{=} \begin{cases}\mathrm{c}_{(H(\varphi) \backslash \varrho(j))}^{\mathfrak{A}} \mathrm{k}_{j}^{\mathfrak{B}^{+}} & \text {if } \mathbf{R}_{j} \in \varphi, \\ 0^{\mathfrak{A}} & \text { otherwise. }\end{cases}
$$

Note that $\mathrm{k}_{j}^{\mathfrak{B}^{+}} \in C l_{\mathbb{Z} \backslash \omega} \mathfrak{B}=A$ by $\left(\mathrm{C}_{8}\right)$, so this definition is meaningful. It is also quite clear that $\boldsymbol{A} \vDash \mathrm{c}_{i} v_{j}=v_{j}[V]$ if $\mathbf{R}_{j} \in \varphi$ and $i \in H(\varphi) \backslash \varrho(j)$. So the proof is complete if we show that $\left(\tau \mu^{\prime} \varphi\right)^{\mathfrak{A}}[V]=F(\varphi)^{\mathfrak{B}^{+}}$. But here one can repeat the proof of the corresponding claim of the $(\Rightarrow)$ part.

In [HMT], Part II, p. 264 (item (2)), it is described how to cut down the number of basic operations of RCA $\omega$ 's to finitely many ones. What follows is an adaptation of that construction to the permissiveness of Task $(*)$.

DEFINITION 2.5.

$$
\mathfrak{A}=\langle A,+,-, 1, \mathrm{c}, \mathrm{d}, \mathrm{e}, \overline{\mathrm{c}}, \text { suc, pred, } \mathrm{k}\rangle \in \mathrm{SCA}
$$

if 1 , e are 0 -ary,,$- \overline{\mathrm{c}}$, suc, pred, $\mathrm{k}$ are unary, $+, \mathrm{c}, \mathrm{d}$ are binary operations and $\mathfrak{A}$ is a model of the following equations (where $0 \stackrel{\text { def }}{=}-1, x \cdot y \stackrel{\text { def }}{=}-(-x+-y)$ and $\oplus$ denotes symmetric difference):

$\left(\mathrm{D}_{0}\right) \quad$ Boolean axioms,

$\left(\mathrm{D}_{1}\right) \quad \mathrm{c}(z, 0)=0$,

$\left(\mathrm{D}_{2}\right) \quad x \leq \mathrm{c}(z, x)$

$\left(\mathrm{D}_{3}\right) \quad \mathrm{c}(z, x \cdot \mathrm{c}(z, y))=\mathrm{c}(z, x) \cdot \mathrm{c}(z, y)$

$\left(\mathrm{D}_{4}\right) \quad \mathrm{c}(z, \mathrm{c}(y, x))=\mathrm{c}(y, \mathrm{c}(z, x))$,

( $\left.\mathrm{D}_{5}\right) \quad \mathrm{d}(z, z)=1$,

$\left(\mathrm{D}_{6}\right) \quad \mathrm{d}(y, z) \cdot \overline{\mathrm{c}}(x \oplus y) \cdot \overline{\mathrm{c}}(x \oplus z)=\mathrm{c}(x, \mathrm{~d}(y, x) \cdot \mathrm{d}(x, z)) \cdot \overline{\mathrm{c}}(x \oplus y) \cdot \overline{\mathrm{c}}(x \oplus z)$,

$\left(\mathrm{D}_{7}\right) \quad \mathrm{c}(y, \mathrm{~d}(y, z) \cdot x) \cdot \mathrm{c}(y, \mathrm{~d}(y, z) \cdot-x) \cdot \overline{\mathrm{c}}(y \oplus z)=0$,

$\left(\mathrm{D}_{8}\right) \quad \overline{\mathrm{c}}(z) \cdot \mathrm{c}(y \cdot \overline{\mathrm{c}}(z), x)=\overline{\mathrm{c}}(z) \cdot \mathrm{c}(y, x)$,

$\left(\mathrm{D}_{9}\right) \quad \overline{\mathrm{c}}(z) \cdot \mathrm{d}(x \cdot \overline{\mathrm{c}}(z), y \cdot \overline{\mathrm{c}}(z))=\overline{\mathrm{c}}(z) \cdot \mathrm{d}(x, y)$,

$\left(\mathrm{D}_{10}\right) \quad \overline{\mathrm{c}}(z) \cdot \operatorname{suc}(\overline{\mathrm{c}}(z) \cdot x)=\overline{\mathrm{c}}(z) \cdot \operatorname{suc}(x)$,

$\left(\mathrm{D}_{11}\right) \quad \overline{\mathrm{c}}(z) \cdot \operatorname{pred}(\overline{\mathrm{c}}(z) \cdot x)=\overline{\mathrm{c}}(z) \cdot \operatorname{pred}(x)$,

$\left(\mathrm{D}_{12}\right) \quad \overline{\mathrm{c}}(z) \cdot \mathrm{k}(\overline{\mathrm{c}}(z) \cdot x)=\overline{\mathrm{c}}(z) \cdot \mathrm{k}(x)$,

$\left(\mathrm{E}_{1}\right) \quad \overline{\mathrm{c}}(0)=0$,

$\left(\mathrm{E}_{2}\right) \quad x \leq \overline{\mathrm{c}}(x)$, 
$\left(\mathrm{E}_{3}\right) \quad \overline{\mathrm{c}}(x \cdot \overline{\mathrm{c}}(y))=\overline{\mathrm{c}}(x) \cdot \overline{\mathrm{c}}(y)$,

$\left(\mathrm{E}_{4}\right) \quad \mathrm{c}(z, \overline{\mathrm{c}}(x))=\overline{\mathrm{c}}(x)$,

$\left(\mathrm{K}_{1}\right) \quad \overline{\mathrm{c}}(\mathrm{e} \cdot-x) \cdot \overline{\mathrm{c}}(-\overline{\mathrm{c}}(x \cdot-\mathrm{e})) \cdot \mathrm{c}(x, \mathrm{k}(y)) \leq \mathrm{k}(y)$,

$\left(\mathrm{S}_{0}\right) \quad x \leq \operatorname{suc}(x)$

$\left(\mathrm{S}_{1}\right) \quad \operatorname{suc}(0)=0$,

$\left(\mathrm{S}_{2}\right) \quad \overline{\mathrm{c}}(\operatorname{suc}(x) \cdot-x) \geq \overline{\mathrm{c}}(x) \cdot \overline{\mathrm{c}}(-x)$,

$\left(\mathrm{S}_{3}\right) \quad \overline{\mathrm{c}}(\mathrm{e})=\overline{\mathrm{c}}(-\mathrm{e})$,

$\left(\mathrm{S}_{4}\right) \quad \operatorname{pred}(\operatorname{suc}(x))=\operatorname{suc}(\operatorname{pred}(x))=x$.

In the above definition $\left(\mathrm{D}_{0}\right)-\left(\mathrm{D}_{7}\right)$ correspond to "ordinary" cylindric axioms, $\left(\mathrm{D}_{8}\right)-\left(\mathrm{D}_{12}\right)$ and $\left(\mathrm{E}_{4}\right)$ make it possible to relativize by a $\overline{\mathrm{c}}$-closed element (cf. Lemma 2.6 below). $\left(\mathrm{E}_{1}\right)-\left(\mathrm{E}_{3}\right)$ say that the $\langle A,+,-, 1, \overline{\mathrm{c}}\rangle$-reduct of $\boldsymbol{A}$ is a $\mathrm{CA}_{1}$. $\left(\mathrm{S}_{0}\right)-\left(\mathrm{S}_{4}\right)$ help us embed $\mathbb{Z}$ into (some) SCA's.

LEMmA 2.6. If $\mathfrak{A} \in \mathrm{SCA}$ is subdirectly irreducible then $0 \neq x \in A$ implies $\overline{\mathrm{c}}(x)=1$.

P r o of. We show that if $a \in A$ is $\bar{c}$-closed then $\mathfrak{A} \cong \boldsymbol{A}\lceil a \times \mathfrak{A} \uparrow-a$. Here $\mathfrak{A} \uparrow a$ is roughly what is called $R l_{a} \mathfrak{A}$ in [HMT], Definition 2.2.1. That is, the universe of $\mathfrak{A}\lceil a$ is $A\lceil a \stackrel{\text { def }}{=}\{x \in A: x \leq a\}$, and if $f$ is a, say, binary function-symbol of the language of SCA then $f^{\mathfrak{A}\lceil a}(x, y)=f^{\mathfrak{A}}(x, y) \cdot a$ for $x, y \in A\lceil a$.

Now suppose that $0<x \in A$ and $a \stackrel{\text { def }}{=} \overline{\mathrm{c}}(x)<1$. It follows from $\left(\mathrm{E}_{1}\right)-\left(\mathrm{E}_{3}\right)$ that both $a$ and $-a$ are $\overline{\mathrm{c}}$-closed. Let $f$ be the function mapping $A$ to $A \uparrow a \times A \uparrow-a$ defined by $f(x)=\langle a \cdot x,-a \cdot x\rangle$. That $f$ is $1-1$ and onto follows from $\left(\mathrm{D}_{0}\right)$. It is also quite easy to show that it respects the operations of $\mathfrak{A}$. For example, let us check c. Since $f(\mathrm{c}(x, y))=\langle a \cdot \mathrm{c}(x, y),-a \cdot \mathrm{c}(x, y)\rangle$ and $\mathrm{c}(f(x), f(y))=$ $\mathrm{c}(\langle a \cdot x,-a \cdot x\rangle,\langle a \cdot y,-a \cdot y\rangle)=\langle a \cdot \mathrm{c}(a \cdot x, a \cdot y),-a \cdot \mathrm{c}(-a \cdot x,-a \cdot y)\rangle$, we have to show $a \cdot \mathrm{c}(x, y)=a \cdot \mathrm{c}(a \cdot x, a \cdot y)$ and $-a \cdot \mathrm{c}(x, y)=-a \cdot \mathrm{c}(-a \cdot x,-a \cdot y)$. We prove the former only, the other one is completely analogous.

$$
\begin{aligned}
a \cdot \mathrm{c}(a \cdot x, a \cdot y) & =a \cdot \mathrm{c}(a \cdot x, \mathrm{c}(a \cdot x, a) \cdot y) \\
& =a \cdot \mathrm{c}(a \cdot x, a) \cdot \mathrm{c}(a \cdot x, y)=a \cdot \mathrm{c}(a \cdot x, y)=a \cdot \mathrm{c}(x, y) .
\end{aligned}
$$

We used $\left(\mathrm{E}_{4}\right),\left(\mathrm{D}_{3}\right),\left(\mathrm{E}_{4}\right)$ again and then $\left(\mathrm{D}_{8}\right)$.

The following lemma is known, but for completeness we include a proof.

LEMMA 2.7. Let $\mathfrak{B}$ be the countable, atomless $\mathrm{BA}$ (note that then $\leq i$ s a dense (partial) ordering). Then there is a $1-1$, onto function $h: B \backslash\{0,1\} \rightarrow B \backslash\{0,1\}$ such that for all $x \in B \backslash\{0,1\}, x<h(x)$.

Proof. Enumerate the elements in $B \backslash\{0,1\}$ as $\left\{b_{n}: n \in \omega\right\}$. Let $i_{0}=$ $\min \left\{i \in \omega: b_{0}<b_{i}\right\}$ and $i_{n+1}=\min \left\{i \in \omega: i \notin\left\{i_{0}, \ldots, i_{n}\right\}, b_{n+1}<b_{i}\right\}$. This definition is meaningful since there are no co-atoms in $\mathfrak{B}$. Let $h\left(b_{n}\right) \stackrel{\text { def }}{=} b_{i_{n}}$ for $n \in \omega$. Since clearly $(\forall x \in B \backslash\{0,1\}) x<h(x)$ and $h$ is $1-1$, it remains to show that it is onto. In other words, we have to check that the function $i: \omega \rightarrow \omega$ is onto. 
Suppose otherwise, and let $k \in \omega$ be such that $(\forall n \in \omega) i_{n} \neq k$. Choose $m$ with $b_{m}<b_{k}$ (there is such an $m$ since $\mathfrak{B}$ is atomless). Let $J=\left\{j>m: b_{m}<b_{j}<b_{k}\right\}$. Then, by density, $|J|=\omega$, so $\left|\left\{i_{j}: j \in J\right\}\right|=\omega$ since $i$ is 1-1. But $\left\{i_{j}: j \in J\right\} \subseteq k$ since for each $j \in J, b_{j}<b_{k}$ and $k \notin\left\{i_{0}, \ldots, i_{j-1}\right\}$ by assumption. Clearly, a contradiction.

DeFinition 2.8. Let $G$ be the following function mapping constant terms of the language of $\mathrm{CA}_{\mathbb{Z}}^{k}$ to constant terms of the language of SCA:

$$
\begin{aligned}
& G\left(\mathrm{k}_{i}\right)=\mathrm{k}\left(\operatorname{suc}^{i}(\mathrm{e})\right) \quad \text { if } i \in \omega, \\
& G(1)=1, \\
& G\left(\mathrm{~d}_{i j}\right)=\mathrm{d}\left(\operatorname{suc}^{i}(\mathrm{e}), \operatorname{suc}^{j}(\mathrm{e})\right) \quad \text { if } i, j \in \mathbb{Z}, \\
& G(\tau+\mu)=G(\tau)+G(\mu), \\
& G(-\tau)=-G(\tau), \\
& G\left(\mathrm{c}_{i} \tau\right)=\mathrm{c}\left(\operatorname{suc}^{i}(\mathrm{e}), G(\tau)\right) \quad \text { if } i \in \mathbb{Z},
\end{aligned}
$$

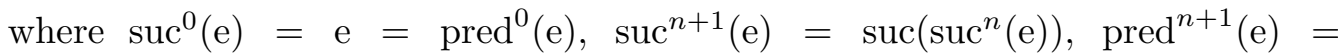
$\operatorname{pred}\left(\operatorname{pred}^{n}(\mathrm{e})\right)$ and $\operatorname{suc}^{-n}(\mathrm{e})=\operatorname{pred}^{n}(\mathrm{e})$ if $n \in \omega$.

Definition 2.9. Define the function $\bar{F}$ on arbitrary first-order formulas (cf. the remark after Definition 2.3) by $\bar{F}(\varphi) \stackrel{\text { def }}{=}(G(F(\varphi))=1)$.

Note that $\bar{F}$ is computable. Moreover, $\bar{F}$ is semantically satisfactory, as will be shown in Theorem 2.11, i.e. after finishing the proof of Theorem 2.0.

LEMMA 2.10. If $\tau$ is a constant $\mathrm{CA}_{\mathbb{Z}}^{k}$-term, then $\mathrm{CA}_{\mathbb{Z}}^{k} \vDash \tau=1$ iff $\mathrm{SCA} \vDash$ $G(\tau)=1$.

Pr o of. $(\Leftarrow)$ Suppose that $\mathrm{CA}_{\mathbb{Z}}^{k} \not \models \tau=1, \tau$ a constant $\mathrm{CA}_{\mathbb{Z}}^{k}$-term. Let $\boldsymbol{A} \in \mathrm{CA}_{\mathbb{Z}}^{k}$ be such that $\tau^{\mathfrak{A}} \neq 1^{\mathfrak{A}}$. We can assume that $\mathfrak{A}$ is subdirectly irreducible.

Case 1. $\mathfrak{A}$ is discrete (in the sense of [HMT], 1.3.10, see also 1.3 .12 op. cit.). We can assume that $\boldsymbol{\mathfrak { A }}$ is finite because of the following: First, assuming $(\forall i \in \omega)(\exists j \in \omega)\left(\mathrm{k}_{j}\right.$ occurs in $\tau$ and $\left.\mathrm{k}_{i}^{\mathfrak{A}}=\mathrm{k}_{j}^{\mathfrak{A}}\right)$ does not make either $\mathfrak{A} \in \mathrm{CA}_{\mathbb{Z}}^{k}$ or $\mathfrak{A} \not \models \tau=1$ false. Then, since $\mathfrak{A}$ is really just a Boolean algebra, the subalgebra generated by those $\mathrm{k}_{i}$ 's which occur in $\tau$ is finite. Let

$$
\mathfrak{B}=\langle B,+,-, 1, \mathrm{c}, \mathrm{d}, \mathrm{e}, \overline{\mathrm{c}}, \text { suc }, \text { pred }, \mathrm{k}\rangle
$$

be an SCA-type algebra defined as follows: $\langle B,+,-, 1\rangle$ is the countable atomless BA. Let $g$ be an embedding of the BA-reduct of $\boldsymbol{A}$ into $\langle B,+,-, 1\rangle$. For $x, y \in B$ let $\mathrm{c}^{\mathfrak{B}}(x, y) \stackrel{\text { def }}{=} y, \mathrm{~d}^{\mathfrak{B}}(x, y) \stackrel{\text { def }}{=} 1, \overline{\mathrm{c}}^{\mathfrak{B}}(0) \stackrel{\text { def }}{=} 0, \overline{\mathrm{c}}^{\mathfrak{B}}(x) \stackrel{\text { def }}{=} 1$ if $x>0, \operatorname{suc}^{\mathfrak{B}}(0) \stackrel{\text { def }}{=} 0$, $\operatorname{suc}^{\mathfrak{B}}(1) \stackrel{\text { def }}{=} 1$ and $\operatorname{suc}^{\mathfrak{B}} \uparrow(B \backslash\{0,1\})$ is the injection onto $B \backslash\{0,1\}$ provided by Lemma 2.7. Let $\operatorname{pred}^{\mathfrak{B}}$ be the inverse of $\operatorname{suc}^{\mathfrak{B}}, 0<\mathrm{e}^{\mathfrak{B}}<1$ and

$$
\mathrm{k}^{\mathfrak{B}}(x) \stackrel{\text { def }}{=} \begin{cases}g\left(\mathrm{k}_{i}^{\mathfrak{A}}\right) & \text { if } \mathrm{k}_{i} \text { occurs in } \tau \text { and } x=\operatorname{suc}^{i}(\mathrm{e}), \\ 0 & \text { otherwise. }\end{cases}
$$


Now it is very easy to check that the SCA-axioms hold in $\mathfrak{B}$. Also, by induction on the subterms of $\tau$ one easily shows that $G(\tau)^{\mathfrak{B}}=g\left(\tau^{\mathfrak{A}}\right) \neq g\left(1^{\mathfrak{A}}\right)=1^{\mathfrak{B}}$, proving $\mathrm{SCA} \not \models G(\tau)=1$.

Case 2. $\mathfrak{A}$ is not discrete. Since $\tau$ is a constant term, we can assume that $\mathfrak{A}$ is generated by the constants, whence it is at most countable and dimension-complemented by [HMT], 2.1.5. It follows from $\mathfrak{A}$ 's being non-discrete and subdirectly irreducible that $\mathfrak{A} \vDash-\mathrm{c}_{i}-\mathrm{d}_{i j}=0$. Thus $\mathfrak{A}$ is atomless by [HMT], 1.11.8.

Fix $m \in \omega$ with $-m<j<m$ for all $j \in O c c(\tau)$, where

$$
\operatorname{Occ}(\tau) \stackrel{\text { def }}{=}\left\{i: \mathrm{c}_{i} \text { or } \mathrm{d}_{i j} \text { or } \mathrm{k}_{i} \text { occurs in } \tau\right\} .
$$

We define an SCA-type algebra

$$
\mathfrak{B}=\langle A,+,-, 1, \mathrm{c}, \mathrm{d}, \mathrm{e}, \overline{\mathrm{c}}, \mathrm{suc}, \text { pred }, \mathrm{k}\rangle,
$$

and show that it is in fact in SCA and $\mathfrak{B} \not \models G(\tau)=1$. Let $+\stackrel{\mathfrak{B}}{\stackrel{\text { def }}{=}}+{ }^{\mathfrak{A}},-\stackrel{\mathfrak{B}}{=} \stackrel{\text { def }}{=}-\mathfrak{A}$, $1^{\mathfrak{B}} \stackrel{\text { def }}{=} 1^{\mathfrak{A}}, \operatorname{suc}^{\mathfrak{B}}(0) \stackrel{\text { def }}{=} 0, \operatorname{suc}^{\mathfrak{B}}(1) \stackrel{\text { def }}{=} 1$ and let $\operatorname{suc}^{\mathfrak{B}} \uparrow(A \backslash\{0,1\})$ be an injection onto $A \backslash\{0,1\}$ with $(\forall x \in A \backslash\{0,1\}) x<\operatorname{suc}(x)$, see Lemma 2.7 above. Let pred $^{\mathfrak{B}}$ be the inverse of $\operatorname{suc}^{\mathfrak{B}}, \mathrm{e}^{\mathfrak{B}} \stackrel{\text { def }}{=} \mathrm{d}_{01}^{\mathfrak{A}}, \overline{\mathrm{c}}^{\mathfrak{B}}(0) \stackrel{\text { def }}{=} 0$ and $\overline{\mathrm{c}}^{\mathfrak{B}}(a) \stackrel{\text { def }}{=} 1$ for all $0<a \in A$.

Let $f: \mathbb{Z} \rightarrow A$ be any bijection with the following properties: $f(i)=\operatorname{suc}^{i}(e)$ if $-m<i<m$ and $f^{\prime \prime}(\mathbb{Z} \backslash \omega)=\{x \in A: x<e\}$. Now let $\mathrm{c}^{\mathfrak{B}}(x, y) \stackrel{\text { def }}{=} \mathrm{c}_{f^{-1} x}^{\mathfrak{A}} y$, $\mathrm{d}^{\mathfrak{B}}(x, y) \stackrel{\text { def }}{=} \mathrm{d}_{f^{-1} x, f^{-1} y}^{\mathfrak{A}}$ and

$$
\mathrm{k}^{\mathfrak{B}}(x) \stackrel{\text { def }}{=} \begin{cases}\mathrm{k}_{f^{-1} x}^{\mathfrak{A}} & \text { if } f^{-1}(x) \geq 0, \\ 0 & \text { otherwise. }\end{cases}
$$

Now we begin checking the axioms in Definition 2.5. $\left(\mathrm{D}_{0}\right)-\left(\mathrm{D}_{7}\right)$ hold in $\mathfrak{B}$ since the corresponding cylindric axioms hold in $\boldsymbol{A}$. To illustrate, let us check $\left(\mathrm{D}_{6}\right)$. If $x=y$ or $x=z$ then both sides of the equation equal 0 . If $x \neq y, z$ then $\overline{\mathrm{c}}(x \oplus y) \cdot \overline{\mathrm{c}}(x \oplus z)=1$ and $f^{-1} x \neq f^{-1} y, f^{-1} z$, so

$$
\begin{aligned}
\text { LHS } & =\mathrm{d}(y, z)=\mathrm{d}_{f^{-1} y, f^{-1} z} \\
& =\mathrm{c}_{f^{-1} x}\left(\mathrm{~d}_{f^{-1} y, f^{-1} x} \cdot \mathrm{d}_{f^{-1} x, f^{-1} z}\right) \\
& =\mathrm{c}(x, \mathrm{~d}(y, x) \cdot \mathrm{d}(x, z))=\mathrm{RHS} .
\end{aligned}
$$

$\left(\mathrm{D}_{8}\right)-\left(\mathrm{D}_{12}\right)$ are satisfied because of the definition of $\overline{\mathrm{c}} .\left(\mathrm{E}_{1}\right)-\left(\mathrm{E}_{3}\right)$ are true in $\mathfrak{B}$ for the same reason. $\left(\mathrm{E}_{4}\right)$ is true since by $\left(\mathrm{D}_{1}\right)-\left(\mathrm{D}_{3}\right)$, both 0 and 1 are "zerodimensional". $\left(\mathrm{K}_{1}\right)$ holds by $\left(\mathrm{C}_{8}\right)$ and the definition of $f$ (note that $\overline{\mathrm{c}}(\mathrm{e} \cdot-x)$. $\overline{\mathrm{c}}(-\overline{\mathrm{c}}(x \cdot-\mathrm{e}))$ is 1 iff $x<\mathrm{e}$, that is, iff $f^{-1} x<0$, and 0 otherwise). Finally, $\left(\mathrm{S}_{0}\right)-\left(\mathrm{S}_{4}\right)$ are easily checked.

Next we claim that $\tau^{\mathfrak{A}}=G(\tau)^{\mathfrak{B}}$. We show this by induction on the subterms of $\tau$. In the proof below $-m<i, j<m$ (recall the definition of $m$ ).

$$
\begin{aligned}
& G\left(\mathrm{k}_{i}\right)^{\mathfrak{B}}=\mathrm{k}\left(\operatorname{suc}^{i}(\mathrm{e})\right)^{\mathfrak{B}}=\mathrm{k}(f(i))^{\mathfrak{B}}=\mathrm{k}_{i}^{\mathfrak{A}} \quad \text { where } 0 \leq i<m, \\
& G(1)^{\mathfrak{B}}=1^{\mathfrak{A}}, \\
& G\left(\mathrm{~d}_{i j}\right)^{\mathfrak{B}}=\mathrm{d}\left(\operatorname{suc}^{i}(\mathrm{e}), \operatorname{suc}^{j}(\mathrm{e})\right)^{\mathfrak{B}}=\mathrm{d}(f(i), f(j))^{\mathfrak{B}}=\mathrm{d}_{i j}^{\mathfrak{A}},
\end{aligned}
$$




$$
\begin{aligned}
& G(\mu+\sigma)^{\mathfrak{B}}=G(\mu)^{\mathfrak{B}}+G(\sigma)^{\mathfrak{B}}=\mu^{\mathfrak{A}}+\sigma^{\mathfrak{A}}=(\mu+\sigma)^{\mathfrak{A}}, \\
& G(-\mu)^{\mathfrak{B}}=-G(\mu)^{\mathfrak{B}}=-\mu^{\mathfrak{A}}=(-\mu)^{\mathfrak{A}}, \\
& G\left(\mathrm{c}_{i} \mu\right)^{\mathfrak{B}}=\mathrm{c}\left(\operatorname{suc}^{i}(\mathrm{e}), G(\mu)\right)^{\mathfrak{B}}=\mathrm{c}^{\mathfrak{B}}\left(f(i), G(\mu)^{\mathfrak{B}}\right)=\mathrm{c}_{i}{ }^{\mathfrak{A}}\left(\mu^{\mathfrak{A}}\right)=\left(\mathrm{c}_{i} \mu\right)^{\mathfrak{A}} .
\end{aligned}
$$

This completes the proof of $(\Leftarrow)$.

$(\Rightarrow)$ Suppose that SCA $\not \models G(\tau)=1$, and let $\mathfrak{B} \in \mathrm{SCA}$ and $V: \omega \rightarrow B$ be such that $G(\tau)^{\mathfrak{B}} \neq 1^{\mathfrak{B}}$. We may suppose that $|B|=\omega$ and $\mathfrak{B}$ is subdirectly irreducible, so Lemma 2.6 applies. Let $A \stackrel{\text { def }}{=} B$ and let $m, f$ be as in the previous part of the proof. Note that the condition " $f(i)=\operatorname{suc}^{i}$ (e) if $-m<i<m$ " is consistent with $f$ being 1-1, i.e. $\left|\left\{\operatorname{suc}^{i}(\mathrm{e}):-m<i<m\right\}\right|=2 m-1$ by Lemma 2.6 and the SCA-axioms $\left(\mathrm{S}_{0}\right)-\left(\mathrm{S}_{4}\right)$. Define the operations of the algebra

$$
\mathfrak{A} \stackrel{\text { def }}{=}\left\langle A,+,-, 1, \mathrm{c}_{i}, \mathrm{~d}_{i j}, \mathrm{k}_{l}\right\rangle_{i, j \in \mathbb{Z}, l \in \omega}
$$

as follows: $+^{\mathfrak{A}} \stackrel{\text { def }}{=}++^{\mathfrak{B}},-{ }^{\mathfrak{A}} \stackrel{\text { def }}{=}-{ }^{\mathfrak{B}}, 1^{\mathfrak{A}} \stackrel{\text { def }}{=} 1^{\mathfrak{B}}, c_{i}^{\mathfrak{A}}(x) \stackrel{\text { def }}{=} \mathrm{c}^{\mathfrak{B}}(f(i), x), \mathrm{d}_{i j}^{\mathfrak{A}} \stackrel{\text { def }}{=}$ $\mathrm{d}^{\mathfrak{B}}(f(i), f(j))$ if $i, j \in \mathbb{Z}$ and $\mathrm{k}_{l}^{\mathfrak{A}}(x) \stackrel{\text { def }}{=} \mathrm{k}^{\mathfrak{B}}(f(l))$ if $l \in \omega$. Using $\left(\mathrm{D}_{0}\right)-\left(\mathrm{D}_{7}\right)$ and $\left(\mathrm{K}_{1}\right)$ it is easy to show that the resulting structure satisfies $\left(\mathrm{C}_{0}\right)-\left(\mathrm{C}_{8}\right)$. Furthermore, one can prove $\tau^{\mathfrak{A}}=G(\tau)^{\mathfrak{B}}$ exactly as in the previous part.

Convention. From now on by a first-order formula we will always mean a restricted one (cf. the remark preceding Definition 2.3).

The semantic aspect of our translation function $\bar{F}$. To see that $\bar{F}$ and SCA are semantically satisfactory, with every model $\mathfrak{M}$ of first-order logic we will associate an SCA $\operatorname{Alg}(\mathfrak{M})$ in a natural way such that for any first-order formula $\varphi$ the meaning of $G(F(\varphi))$ in $\operatorname{Alg}(\mathfrak{M})$ will be the same as the meaning of $\varphi$ in $\mathfrak{M}$. In particular, we will have

$$
(* * *) \quad \mathfrak{M} \vDash \varphi \quad \text { iff } \quad \operatorname{Alg}(\mathfrak{M}) \vDash \bar{F}(\varphi) .
$$

Such a correspondence with $\mathrm{CA}_{\omega}$ in place of SCA was elaborated in detail in [HMT], $\S 4.3$ (and in a style that is as satisfactory as the case of propositional logic was). Since there is no essential difference between $\omega$ and $\mathbb{Z}$ as an index set, we can take the correspondence in $[\mathrm{HMT}], \S 4.3$, as between first-order logic and $\mathrm{CA}_{\mathbb{Z}}$. The only change is that in first-order logic we have to index our variables with integers instead of natural numbers. Then we have the above desired $(* * *)$ for $C A_{\mathbb{Z}}$ in place of SCA. The only changes to do are that we first replace $C A_{\mathbb{Z}}$ with $C A_{\mathbb{Z}}^{k}$ and then $C A_{\mathbb{Z}}^{k}$ with SCA.

In [HMT], 4.3.3-4.3.10, with each model $\mathfrak{M}$ a pair $\langle\mathfrak{C s} \mathfrak{M}, k\rangle$ is associated where $\mathfrak{C s} \mathfrak{M} \in \mathrm{CA}_{\mathbb{Z}}$ and $k$ is an evaluation of the algebraic variables $\left\{v_{i}: i \in \omega\right\}$ into $\mathfrak{C} \mathfrak{s} \mathfrak{M}$. The function $\tau \mu^{\prime}$ mapping first-order formulas to $\mathrm{CA}_{\mathbb{Z}}$-terms is defined in $[\mathrm{HMT}]$, 4.3.60. It is proved there that

$$
\mathfrak{M} \vDash \varphi \quad \text { iff } \quad \mathfrak{C} \mathfrak{s} \mathfrak{M} \vDash \tau \mu^{\prime}(\varphi)=1[k],
$$

moreover,

$$
\varphi^{\mathfrak{M}}=\tau \mu^{\prime}(\varphi)^{\mathfrak{C} \mathfrak{s} \mathfrak{M}}[k]
$$


where $\varphi^{\mathfrak{M}}$ is the set of those valuations $s \in \mathbb{Z}_{M}$ such that $\mathfrak{M} \vDash \varphi[s]$ and $\tau \mu^{\prime}(\varphi)^{\mathfrak{C} \mathfrak{s} \mathfrak{M}}[k]$ is the value of the term $\tau \mu^{\prime}(\varphi)$ in the algebra $\mathfrak{C} \mathfrak{s} \mathfrak{M}$ at the evaluation $k$ of the variables in $\tau \mu^{\prime}(\varphi)$.

Now our $F$ differs from $\tau \mu^{\prime}$ only in that the evaluation $k$ is built into $F$. That is, with the atomic formula $\mathbf{R}_{j} v_{0} \ldots v_{\varrho(j)-1}$ we associate the constant symbol $\mathrm{k}_{j}$ instead of the algebraic variable $v_{j}$ (as was done in the definition of $\left.\tau \mu^{\prime}\right)\left({ }^{8}\right.$ ). Then by (4) we have

$$
\mathfrak{M} \vDash \varphi \quad \text { iff } \quad\langle\mathfrak{C} \mathfrak{s} \mathfrak{M}, k\rangle \vDash F(\varphi)=1,
$$

and similarly for $\left(4^{+}\right)$, that is, if we denote $\langle\mathfrak{C s} \mathfrak{M}, k\rangle$ by $\operatorname{Al}(\mathfrak{M})$, then $\operatorname{Al}(\mathfrak{M}) \in \mathrm{CA}_{\mathbb{Z}}^{k}$ and we have

$$
\varphi^{\mathfrak{M}}=F(\varphi)^{\mathrm{Al}(\mathfrak{M})} .
$$

Now, (5) is a statement of the desired (***)-type, the only problem is that it acts between first-order logic and $\mathrm{CA}_{\mathbb{Z}}^{k}$ instead of SCA. But we have already seen (in the proof of Lemma 2.10) that it is easy to commute between $C A_{\mathbb{Z}}^{k}$ and SCA. So let us do it. First assume that $\mathfrak{M}$ is nontrivial, i.e. it has more than one element. The case of trivial $\mathfrak{M}$ is easy (as in the proof of Lemma 2.10) so let us come back to it later. Since $\operatorname{Al}(\mathfrak{M})$ is generated by $\left\{\mathrm{k}_{i}: i \in \omega\right\}$ (and $\mathfrak{M}$ is nontrivial) we have that $\mathrm{Al}(\mathfrak{M})$ is countable and atomless (cf. [HMT], §4.3, especially Remark 4.3.11). Then, by the proof of Lemma $2 \cdot 10(\Leftarrow)$, we can add suc, pred, e and $\bar{c}$ to $\mathrm{Al}(\mathfrak{M})$ (and of course change $\mathrm{c}_{i}(x)$ to $\mathrm{c}\left(\operatorname{suc}^{i}(\mathrm{e}), x\right)$ etc.) to obtain a $\mathfrak{B} \in \mathrm{SCA}$ such that the $\mathrm{CA}_{\mathbb{Z}}^{k}$-type reduct (in the obvious sense) of $\mathfrak{B}$ is exactly $\operatorname{Al}(\mathfrak{M})$. Let $\operatorname{Alg}(\mathfrak{M})$ be defined to be this $\mathfrak{B}$. It was proved in Lemma 2.10 that $F(\varphi)^{\mathrm{Al}(\mathfrak{M})}=G(F(\varphi))^{\operatorname{Alg}(\mathfrak{M})}$ (there it was proved for arbitrary constant $\mathrm{CA}_{\mathbb{Z}}^{k}$-terms in place of $F(\varphi)$ ). Thus (5) yields

$$
\mathfrak{M} \vDash \varphi \quad \text { iff } \quad \operatorname{Alg}(\mathfrak{M}) \vDash G(F(\varphi))=1,
$$

which is of course just a special case of

$$
\varphi^{\mathfrak{M}}=G(F(\varphi))^{\mathrm{Alg}(\mathfrak{M})},
$$

given by $\left(5^{+}\right)$. But this not only proves $(* * *)$ but also improves it since our $\bar{F}$ is defined as $\bar{F}=F \circ G$.

Let us turn to the trivial models. Assume $\mathfrak{M}$ is trivial. Then $\operatorname{Al}(\mathfrak{M})$ has two elements, $\emptyset$ and ${ }^{\mathbb{Z}} M$. It is easy to construct a countable atomless $\mathfrak{C} \in \mathrm{CA}_{\mathbb{Z}}^{k}$ such that $\mathfrak{A} \subseteq \mathfrak{C}$ (hint: let $\mathrm{c}_{i}$ be the identity function on $\mathfrak{C}$ for all $i$ ). Exactly as we did in the case of nontrivial $\mathfrak{M}$, we associate with $\mathfrak{C}$ a $\mathfrak{B}$ in SCA such that the $\mathrm{CA}_{\mathbb{Z}}^{k}$-reduct of $\mathfrak{B}$ is $\mathfrak{C}$. We let $\operatorname{Alg}(\mathfrak{M}) \stackrel{\text { def }}{=} \mathfrak{B}$. The rest of the proof is now exactly as in the previous case. So we have proved (6) for the case when $\mathfrak{M}$ is trivial.

Thus we have proved the following

THEOREM 2.11. (i) Alg defined above is a natural function mapping models of first-order logic into SCA such that for any formula $\varphi$ and model $\mathfrak{M}$ the meaning

$\left({ }^{8}\right)$ Actually, our $F$ contains slightly more information, but that is irrelevant now. 
of $\varphi$ in $\mathfrak{M}$ is the same as the meaning of $F(G(\varphi))$ in $\operatorname{Alg}(\mathfrak{M})$, that is, $\left(6^{+}\right)$above holds. Moreover,

(ii) $\operatorname{Alg}(\mathfrak{M})$ consists of the meanings $\varphi^{\mathfrak{M}}$ of formulas $\varphi$ in $\mathfrak{M}$, provided $\mathfrak{M}$ is nontrivial.

Theorems 2.0, 2.11 together provide a solution to Task $(*)$ together with condition $(* *)$. (It is Theorem 2.11 which proves that Condition $(* *)$ is satisfied by SCA, $\bar{F}$ (or $F \circ G$ ) and Alg.)

Now we want to outline why this solution does not solve the finitization problem. Since we mentioned only one concrete form of the problem $\left({ }^{9}\right)$, we must be content with showing that (the logic corresponding to) SCA is not a solution of that version. But we hope that this will convince the reader that SCA is not even a candidate for being the variety the finitization problem asks for.

Let $L_{1}$ be a logic such that the algebraic counterpart (in the sense fixed in Problem 0 ) is (a subclass of) SCA. In $L_{1}$ we have logical constants corresponding to operations of SCA; it is not quite clear what meaning we should give to the logical constants $\overline{\mathrm{c}}$, suc, pred and e but suppose that we have given some.

CLAIM. There is an algebra in SCA which is not isomorphic to the semantical Lindenbaum-Tarski algebra of any theory of $L_{1}$.

The reason is that the Lindenbaum-Tarski algebra $\mathfrak{B} \in$ SCA of the theory of the model $\mathfrak{M}=\langle 2, \emptyset, \ldots, \emptyset, \ldots\rangle$ (i.e. the theory of the two-element model with empty relations) can be easily changed to a $\mathfrak{B}^{\prime} \in \mathrm{SCA}$ such that $\mathfrak{B}^{\prime}$ is not isomorphic to $\mathfrak{B}$ but still "says" that in the class $\mathcal{K}$ of models (if any) of which it is the Lindenbaum-Tarski algebra, all models have two-element universes and empty relations. (One way to achieve this is to change the meaning of e so that $0<\mathrm{e}^{\mathfrak{B}^{\prime}}<1$ but the dimension set of $\mathrm{e}^{\mathfrak{B}^{\prime}}$ is different from that of $\mathrm{e}^{\mathfrak{B}}$. We have at least the infinite set $\left\{\mathrm{d}\left(\operatorname{suc}^{i} \mathrm{e}, \operatorname{suc}^{j} \mathrm{e}\right)^{\mathfrak{B}}: i<j \in \mathbb{Z}\right\} \backslash\left\{\mathrm{e}^{\mathfrak{B}}\right\}$ available to choose from.) So, by the isomorphism axiom of Abstract Model Theory (which says that isomorphic models have identical theories) $\mathfrak{B}^{\prime}$ is the Lindenbaum-Tarski algebra of the theory of $\mathfrak{M}$, contradicting $\mathfrak{B} \neq \mathfrak{B}^{\prime}$. This more or less proves the Claim, and it is not hard to push this proof a little further and show that $\mathbf{B}^{\prime}$ is not even in the quasivariety generated by the Lindenbaum-Tarski algebras of the semantical theories of $L_{1}$

We would like to stress that it is not outright impossible to give a solution to Task $(*)$ which happens to solve (some version $\left({ }^{10}\right)$ of) the finitization problem too, but this of course does not change the fact that Task $(*)$ even together with $(* *)$ is not the right question.

As a curiosity, we would like to know how difficult that stronger version of Task $(*)$ is, where we require the following "two way" version of $(* *)$ : In addition to $(* *)$ we require every subdirectly irreducible member of $V$ to be of the form

\footnotetext{
$\left({ }^{9}\right)$ But see Appendix A.
}

$\left({ }^{10}\right)$ E.g. Problem 0 above. 
$\operatorname{Alg}(\mathfrak{M})$ (up to isomorphism), or at least that $\{\operatorname{Alg}(\mathfrak{M}): \mathfrak{M}$ is a model $\}$ generates $V$ as a quasivariety or variety. This is still not an open problem, because Sain [S87] contains a solution for this stronger "two-way" version of Task $(*)$ with $(* *)$, but we are curious whether this task still admits easy solutions like the ones in the present paper.

We note that the present work is part of a research project jointly pursued by Hajnal Andréka and the author. E.g. more information on the purely logical form of the finitization problem will be available from them; till then the reader is referred (for this logical form) to Appendix A below and $\S 4$ of Sain [S87], Simon [S90], Venema [V90], [V92].

\section{Appendix A}

Here we give some concrete instances of the finitization problem (most of which are important variants of Problem 0). From now on FOL abbreviates first-order logic.

PROBLEM 1. Is there a stronger (or equivalent) formulation $L_{1}$ of FOL such that the class $\operatorname{LT}\left(L_{1}\right)$ of all semantical Lindenbaum-Tarski algebras of theories of $L_{1}$ forms a finitely axiomatizable variety or quasivariety (up to isomorphism, of course)? Here $\operatorname{LT}\left(L_{1}\right)$ is defined in Problem 0. Further, in the present problem it is permitted to add infinitary predicate symbols to $L_{1}$ in the style of [HMT], $\S 4.3$ (where it was shown that this does not diminish relevance of $L_{1}$ to FOL $\left({ }^{11}\right)$ ). Of course, the semantics (i.e. evaluation of variables, satisfaction, etc.) of the new infinitary atomic formulas $\mathbf{R}\left(v_{0} \ldots v_{n} \ldots\right)_{n \in \omega}$ remains the old one.

Problem 1 is harder than Problem 0 since here $\operatorname{LT}\left(L_{1}\right)$ itself has to be a (finitely axiomatizable) quasivariety.

Problem 2. Find a formulation $L_{1}$ of FOL related to FOL exactly as described in Problem 1 above (that is, $L_{1}$ is stronger than or equivalent to FOL and $L_{1}$ is permitted to have infinitary predicates) such that $L_{1}$ admits a strongly complete Hilbert-style axiomatization $\vdash_{1}$ of the following kind:

$\vdash_{1}$ is given by finitely many axiom schemas, where axiom schemas are built up from formula variables $\Psi_{1}, \ldots, \Psi_{n}, \ldots$ (ranging over formulas of $L_{1}$ ) by the logical connectives of $L_{1}$. That is, $S c h$, the set of schemas, is the smallest set containing the formula variables and such that for every $n$-ary connective $C$ of $L_{1}$, if $A_{1}, \ldots, A_{n} \in S c h$ then $C\left(A_{1}, \ldots, A_{n}\right) \in S c h$. Only elements of $S c h$ are

$\left.{ }^{(11}\right)$ The infinitary predicates (or atomic formulas) are needed to remove the following trivial (and slightly irrelevant) reason for nonaxiomatizability of $\operatorname{LT}\left(L_{\omega \omega}\right)$. The atomic formulas of $L_{\omega \omega}$ may have arbitrarily big but finite ranks. Therefore in some ultraproducts of members of $\operatorname{LT}\left(L_{\omega \omega}\right)$ there will be an element corresponding to some "imaginary formula" of infinite rank. Cf. [HMT], §4.3, and Németi [N89]. 
allowed as axiom schemas for $\vdash_{1}$. So, $\vdash_{1}$ is given by finitely many elements of $S c h$ together with modus ponens and the "congruence" rules

$$
\left\{\left(\Psi_{1} \leftrightarrow \Psi_{n+1}\right), \ldots,\left(\Psi_{n} \leftrightarrow \Psi_{n+n}\right)\right\} \vdash_{1} C\left(\Psi_{1}, \ldots, \Psi_{n}\right) \leftrightarrow C\left(\Psi_{n+1}, \ldots, \Psi_{n+n}\right)
$$

for every $n$-ary connective $C$ of $L_{1}$.

A slightly more permissive but still very interesting version of the problem allows $\vdash_{1}$ to be presented by finitely many axiom and rule schemas. Here a rule schema is of the form $A_{1}, \ldots, A_{n} \vdash_{1} A_{0}$, where $\left\{A_{i}: i \leq n\right\} \subseteq S c h$ and for each instance $\varphi_{1}, \ldots, \varphi_{n} \vdash_{1} \varphi_{0}$ of this schema (where the $\varphi_{i}$ 's are concrete formulas of $\left.L_{1}\right)$ we require $\varphi_{1}, \ldots, \varphi_{n} \vDash_{1} \varphi_{0}$.

Note that in Problem 2 above

$$
\Psi_{0} \rightarrow \forall x \Psi_{0} \text { if } x \text { does not occur in } \Psi_{0}
$$

is not a permitted axiom schema. Also,

$$
\exists x \Psi_{0} \vdash_{1} \Psi_{0} \text { if } x \text { does not occur in } \Psi_{0}
$$

is not a rule schema. Further, $\forall x \Psi_{0}(x) \vdash_{1} \Psi_{0}(x / y)$, where " $(x / y)$ " denotes substitution of $y$ for $x$, is not a rule schema either. By contrast, $\forall x \Psi_{0} \vdash_{1} \exists x(x=$ $\left.y \wedge \Psi_{0}\right)$ has the same meaning and it is a rule schema. As a final illustration, $\forall x \forall y(x=y) \vdash_{1} x \neq x$ is not a sound rule schema although it yields logical validities whenever applied to logical validities (but $\forall x \forall y(x=y) \not \nvdash_{1} x \neq x$ ).

An earlier version of this problem asked for a (not necessarily strongly) complete axiomatization, but in view of Sain's result quoted below only the above stronger version remains open.

In connection with Problem 2 above we note that $L_{\omega \omega}$ does not admit such "finite schematic" complete axiomatization as required there. One reason is that $L_{\omega \omega}$ has infinitely many logical connectives (e.g. " $\exists v_{i}$ " for each $i \in \omega$ ). There is, however, a deeper reason. Namely, the obvious ways of cutting down the number of connectives $\left({ }^{12}\right)$ do not help, cf. e.g. [N91], [S87], [HMT], §4.3. On the other hand, propositional logic as well as propositional modal logics are axiomatizable in the style required in Problem 2.

For FOL without equality there is an $L_{1}$ presented in $\S 4$ of Sain [S87] which solves Problem 2 (for the case without equality). This solution also gives rise to a further open problem. Namely, Sain's logic $L_{1}$ (while completely satisfying the conditions of Problem 2) is somewhat complicated. Therefore it remains an important problem to simplify Sain's $L_{1}$. For logic with equality, [S87], together with [S87a], presents a choice of $L_{1}$ with a weakly complete $\vdash_{1}$ (satisfying all the other requirements in Problem 2) and proves that there is no strongly complete $\vdash_{1}$ for this $L_{1}$.

$\left({ }^{12}\right)$ E.g. taking a finite variable fragment $L_{n}$, or coding the infinity of old connectives with finitely many new ones (cf. [HMT], Part II, p. 264, item (2)). 
Consider Problem 0 now. If we replace $\operatorname{Alg}\left(L_{1}\right)$ there with the variety generated by $\operatorname{LT}\left(L_{1}\right)$ then [S87] contains positive solutions for FOL both with and without equality.

Next we formulate a slightly easier but equally important version of Problem 0.

Problem 3. Let $\operatorname{Alg}\left(L_{1}\right)$ be defined as in Problem 0. Recall that Problem 0 requires $\operatorname{Alg}\left(L_{1}\right)$ to be a finitely axiomatizable variety. Now keep the condition that $\operatorname{Alg}\left(L_{1}\right)$ is finitely axiomatizable but drop the requirement that it is a variety. Leave all the rest of Problem 0 unchanged. Is there a positive solution for this slightly weaker problem?

\section{Appendix B}

Here we give a detailed solution for Task $(*)$ based entirely on results proved in $[D]$ for the reader not convinced by the proof presented in $\S 1$. Accordingly, we will use the notation of $[\mathrm{D}]$ extensively.

Let $M$ be a Turing machine with instructions $q_{0}, \ldots, q_{n}$ such that given the first-order formula $\varphi$ as input (that is, starting at instruction $q_{0}$ and scanning the leftmost symbol of $\ulcorner\varphi\urcorner) M$ eventually halts iff $\vDash \varphi$. Let $\Pi(M)$ be the semi-Thue process on the alphabet $A=\left\{0,1, q_{0}, \ldots, q_{n}, q, q^{\prime}, h\right\}$ corresponding to $M$ and defined on pp. 572-574 of [D]. We do not have to worry about the details, all that is needed is

$$
M \text { eventually halts at the input } x \text { iff } h q_{0}\ulcorner x\urcorner h \sim h q^{\prime} h,
$$

which is an immediate corollary of [D], Theorem 2.4 and Lemma 3.2, and

$$
\text { if } g \rightarrow g^{\prime} \in \Pi(M) \text {, then } g \neq \Lambda \neq g^{\prime} \text {. }
$$

Here $\sim \stackrel{\text { def }}{=} \underset{\bar{\Pi}(M)}{\sim}$ and $\Lambda$ is the empty word.

Let $t$ be the similarity type $\left\{\langle\cdot, 2\rangle,\langle a, 0\rangle_{a \in A}\right\}$. Define the function $\beta$ mapping constant (i.e. variable-free) t-terms onto $A^{*} \backslash\{\Lambda\}$ by $\beta(a) \stackrel{\text { def }}{=} a$ if $a \in A$ and $\beta(\tau \cdot \sigma) \stackrel{\text { def }}{=} \beta(\tau) \beta(\sigma)$ (i.e. the concatenation of $\beta(\tau)$ and $\beta(\sigma))$. Let

$$
\begin{aligned}
A x \stackrel{\text { def }}{=} & \left\{\left(x_{1} \cdot x_{2}\right) \cdot x_{3}=x_{1} \cdot\left(x_{2} \cdot x_{3}\right)\right\} \\
& \cup\{\tau=\sigma: \tau, \sigma \text { are constant } t \text {-terms and } \beta(\tau) \rightarrow \beta(\sigma) \in \Pi(M)\} .
\end{aligned}
$$

Then $A x$ is a bit redundant but still finite. Note that by associativity

$$
\beta(\tau)=\beta(\sigma) \Rightarrow A x \vdash \tau=\sigma .
$$

Let $V \stackrel{\text { def }}{=} \operatorname{Mod}(A x)$. Theorem B.0 below says that this is the finitely based variety we are after. Define the (easily computable) function $G$ on first-order formulas by $G(\varphi) \stackrel{\text { def }}{=} \tau$ iff $\tau$ is the constant $t$-term (with, say, the smallest code according to 
some fixed Gödel numbering) such that $\beta(\tau)=h q_{0}\ulcorner\varphi\urcorner h$ and let $F(\varphi) \stackrel{\text { def }}{=}(G(\varphi)=$ $\left.\left(h \cdot q^{\prime}\right) \cdot h\right)$.

Theorem B.0. $V$ is a finitely axiomatizable variety, $F$ is computable and for every first-order formula $\varphi$

$$
\vDash \varphi \Leftrightarrow V \vDash F(\varphi) .
$$

This is an immediate consequence of what we have said so far and of Lemma B.2 below.

Lemma B.1. Let $\tau, \sigma$ be t-terms with $\operatorname{Var}(\tau \cdot \sigma) \subseteq\left\{x_{1}, \ldots, x_{k}\right\}$, and let $\mu_{1}, \ldots, \mu_{k}$ be constant t-terms. Suppose that $A x \vdash \tau=\sigma$. Then $\beta \tau\left(\mu_{i} / x_{i}\right) \sim$ $\beta \sigma\left(\mu_{i} / x_{i}\right)$.

Here $\tau\left(\mu_{i} / x_{i}\right)$ denotes the result of simultaneously substituting $\mu_{i}$ for $x_{i}(i=$ $1, \ldots, k)$ in $\tau$.

Proof. By induction on the derivation of $\tau=\sigma$ from $A x$. If $\tau=\sigma$ is the associativity axiom then $\beta \tau\left(\mu_{i} / x_{i}\right)=\beta\left(\mu_{1}\right) \beta\left(\mu_{2}\right) \beta\left(\mu_{3}\right)=\beta \sigma\left(\mu_{i} / x_{i}\right)$. If it is some other axiom then $\operatorname{Var}(\tau \cdot \sigma)=\emptyset$ and so $\beta \tau\left(\mu_{i} / x_{i}\right)=\beta(\tau) \sim \beta(\sigma)=\beta \sigma\left(\mu_{i} / x_{i}\right)$ since $\beta(\tau) \rightarrow \beta(\sigma) \in \Pi(M)$.

Since $\sim$ is an equivalence relation, we only have to check the derivation rules "substitution" and "replacement".

First suppose that $A x \vdash \tau^{\prime}=\sigma^{\prime}$ and $\tau=\tau^{\prime}(\mu / x), \sigma=\sigma^{\prime}(\mu / x)$, where $\mu$ is some (not necessarily constant) t-term. We may suppose that $x \in \operatorname{Var}\left(\tau^{\prime} \cdot \sigma^{\prime}\right)$. Then $\operatorname{Var}(\mu) \subseteq\left\{x_{1}, \ldots, x_{k}\right\}$ whence $\mu^{\prime} \stackrel{\text { def }}{=} \mu\left(\mu_{i} / x_{i}\right)$ is a constant $t$-term. Since $\operatorname{Var}\left(\tau^{\prime} \cdot \sigma^{\prime}\right) \subseteq\left\{x, x_{1}, \ldots, x_{k}\right\}$, the induction hypothesis gives

$$
\begin{aligned}
\beta\left(\tau\left(\mu_{i} / x_{i}\right)\right) & =\beta\left(\left(\tau^{\prime}(\mu / x)\right)\left(\mu_{i} / x_{i}\right)\right)=\beta\left(\tau^{\prime}\left(\mu^{\prime} / x, \mu_{i} / x_{i}\right)\right) \\
& \sim \beta\left(\sigma^{\prime}\left(\mu^{\prime} / x, \mu_{i} / x_{i}\right)\right)=\beta\left(\left(\sigma^{\prime}(\mu / x)\right)\left(\mu_{i} / x_{i}\right)\right)=\beta\left(\sigma\left(\mu_{i} / x_{i}\right)\right) .
\end{aligned}
$$

For replacement, suppose that $\tau=\tau_{1} \cdot \tau_{2}, \sigma=\sigma_{1} \cdot \sigma_{2}$ and $A x \vdash \tau_{j}=\sigma_{j}, j=1,2$. Then $\operatorname{Var}\left(\tau_{j} \cdot \sigma_{j}\right) \subseteq\left\{x_{1}, \ldots, x_{k}\right\}$ so the induction hypothesis gives $\beta \tau_{j}\left(\mu_{i} / x_{i}\right) \sim$ $\beta \sigma_{j}\left(\mu_{i} / x_{i}\right)$ for $j=1,2$. But then $\beta\left(\tau\left(\mu_{i} / x_{i}\right)\right)=\beta\left(\tau_{1}\left(\mu_{i} / x_{i}\right)\right) \beta\left(\tau_{2}\left(\mu_{i} / x_{i}\right)\right) \sim$ $\beta\left(\sigma_{1}\left(\mu_{i} / x_{i}\right)\right) \beta\left(\sigma_{2}\left(\mu_{i} / x_{i}\right)\right)=\beta\left(\sigma\left(\mu_{i} / x_{i}\right)\right)$ by the definition of $\sim$.

LEMMA B.2. If $\tau, \sigma$ are constant $t$-terms then

$$
\beta(\tau) \sim \beta(\sigma) \Leftrightarrow A x \vdash \tau=\sigma .
$$

Proof. $(\Rightarrow)$ If $\beta(\tau) \sim \beta(\sigma)$ then there are $u_{0}, \ldots, u_{k} \in A^{*}$ and productions $P_{1}, \ldots, P_{k}$ in $\bar{\Pi}(M)$ such that

$$
\beta(\tau)=u_{0} \Rightarrow_{P_{1}} u_{1} \Rightarrow_{P_{2}} \ldots \Rightarrow_{P_{k}} u_{k}=\beta(\sigma) .
$$

So it is enough to show that $A x \vdash \mu=\mu^{\prime}$ whenever $\beta(\mu)=v g w, \beta\left(\mu^{\prime}\right)=v g^{\prime} w$ for some $v, w \in A^{*}$, and $g \rightarrow g^{\prime} \in \Pi(M)$.

If $v=\Lambda=w$ then $\mu=\mu^{\prime} \in A x$. By (2) there are constant $t$-terms $\varrho, \varrho^{\prime}$ with $\beta(\varrho)=g, \beta\left(\varrho^{\prime}\right)=g^{\prime}$. Note that $\varrho=\varrho^{\prime}$ is an axiom. Now suppose that $v \neq \Lambda=w$ and let $\nu$ be a constant $t$-term with $\beta(\nu)=v$. Then $\beta(\nu \cdot \varrho)=v g$ and $\beta\left(\nu \cdot \varrho^{\prime}\right)=v g^{\prime}$ 
so by (3) it is enough to see $A x \vdash \nu \cdot \varrho=\nu \cdot \varrho^{\prime}$. But that is true because of the replacement rule of equational logic.

The remaining cases (i.e. $v=\Lambda \neq w$ and $v \neq \Lambda \neq w$ ) are treated similarly.

$(\Leftarrow)$ This direction follows from Lemma B.1.

Proof of Theorem B.0. $\vDash \varphi$ iff $M$ halts if it is given the input $\varphi$ iff $h q_{0}\ulcorner\varphi\urcorner h \sim h q^{\prime} h$. By Lemma B.2, this is equivalent to $A x \vdash G(\varphi)=\left(h \cdot q^{\prime}\right) \cdot h$, that is, to $V \vDash F(\varphi)$.

\section{References}

[A91] H. Andréka, Complexity of the equations valid in algebras of relations, thesis for D.Sc. (a post-habilitation degree), Hungar. Acad. Sci., Budapest 1991.

[AMN] H. Andréka, J. D. Monk and I. Németi (eds.), Algebraic Logic (Proc. Conf. Budapest 1988), Colloq. Math. Soc. János Bolyai 54, North-Holland, Amsterdam 1991.

[ANS] H. Andréka, I. Németi and I. Sain, Abstract model-theoretic approach to algebraic logic, manuscript, 1984.

[BF] J. Barwise and S. Feferman (eds.), Model-Theoretic Logics, Springer, Berlin 1985.

[BMP] C. H. Bergman, R. D. Maddux and D. L. Pigozzi (eds.), Algebraic Logic and Universal Algebra in Computer Science, Lecture Notes in Comput. Sci. 425, Springer, Berlin 1990.

[BP] W. J. Blok and D. Pigozzi, Algebraizable logics, Mem. Amer. Math. Soc. 396 (1989).

[B86] P. Burmeister, A model-theoretic oriented approach to partial algebras, AkademieVerlag, Berlin 1986.

[BS] S. Burris and H. P. Sankappanavar, A Course in Universal Algebra, Springer, New York 1981

[CV] W. Craig and R. L. Vaught, Finite axiomatizability using additional predicates, J. Symbolic Logic 23 (3) (1958), 289-308.

[D] M. Davis, Unsolvable Problems, in: Handbook of Mathematical Logic, J. Barwise (ed.), North-Holland, Amsterdam 1977, 567-594.

$[\mathrm{H}] \quad$ L. Henkin, The representation theorem for cylindric algebras, in: Mathematical Interpretations of Formal Systems, North-Holland, Amsterdam 1955, 85-97.

[HM] L. Henkin and J. D. Monk, Cylindric algebras and related structures, in: Proceedings of the Tarski Symposium, Amer. Math. Soc., 1974, 105-121.

[HMT] L. Henkin, J. D. Monk and A. Tarski, Cylindric Algebras, Parts I, II, NorthHolland, Amsterdam 1971, 1985.

[M] R. Maddux, Finitary algebraic logic, Z. Math. Logik Grundlag. Math. 35 (1989), 321-332.

[M70] J. D. Monk, On an algebra of sets of finite sequences, J. Symbolic Logic 35 (1970), $19-28$.

[MLn] - Lectures on cylindric set algebras, this volume.

[N89] I. Németi, On cylindric algebraic model theory, in [BMP], 37-75.

[N91] -, Algebraization of quantifier logics: an introductory overview, Studia Logica 4 (1991), in press.

[S87] I. Sain, Searching for a finitizable algebraization of first order logic, submitted, 1987.

[S87a] -, Positive results related to the Jónsson, Tarski-Givant representation problem, preprint, Math. Inst. Hungar. Acad. Sci., Oct. 1987.

[ST] I. Sain and R. J. Thompson, Strictly finite schema axiomatization of quasi-polyadic algebras, in [AMN], 539-571. 
[S90] A. Simon, A complete calculus for type-free logic and representable cylindric algebras, preprint, Math. Inst. Hungar. Acad. Sci.

[S91] - Finite schema completeness for typeless logic and representable cylindric algebras, in $[\mathrm{AMN}], 665-670$

[TG] A. Tarski and S. Givant, A Formalization of Set Theory without Variables, Colloq. Publ. 41, Amer. Math. Soc., 1986.

[VH] P. A. S. Veloso and A. M. Haeberer, A finitary relational algebra for classical first order logic, preprint, Rio de Janeiro; abstracted in: Bull. Section of Logic 20 (2) (1991), $52-62$.

[V90] Y. Venema, Cylindric modal logic, submitted to J. Symbolic Logic.

[V92] —, Many-dimensional modal logic, Ph.D. thesis, Univ. of Amsterdam, 1992. 